\title{
Fatal Rhabdomyolysis following Spine Surgery in a Morbidly Obese Patient: A Case Report
}

\author{
Se Ho Jung ${ }^{1}$, Seok Won $\mathrm{Kim}^{1}$, Dong Min $\mathrm{Kim}^{2}$, Chang II Ju${ }^{1}$ \\ Departments of ${ }^{I}$ Neurosurgery, ${ }^{2}$ Internal Medicine, Chosun University College of Medicine, Gwangju, Korea
}

We generally believe that obese patients are faced on higher risk of developing perioperative complications. Rhabdomyolysis is a rare but potentially life-threatening condition caused by the release of injured skeletal muscle components into the circulation. It usually results from mechanical damage to the muscle, intoxication, or a postictal state after a seizure. In the present study, we have reported a rare case of rhabdomyolysis developing in a morbidly obese patient after upper thoracic spinal fusion surgery. We have found acute renal failure that evolved during the course of treatment resulted in a fatal outcome even though the patient received intensive supportive care. Our experience suggests that this rare complication should be considered in morbidly obese patients and those efforts should be made to avoid rhabdomyolysis.

Key Words: Spine $\cdot$ Rhabdomyolysis • Obesity

\section{INTRODUCTION}

Rhabdomyolysis causes muscle breakdown and the leakage of intracellular muscle constituents, such as enzymes, electrolytes, and myoglobin into the circulation and extracellular fluid $^{3,6,8)}$. It has been described as a clinical syndrome initially characterized by darker urine, muscle pain, and weakness from prolonged muscle compression, as seen in intoxicated individuals or in individuals after status epilepticus ${ }^{10,11)}$. Multisystem trauma, the static position of an intoxicated patient, or the postictal period after a seizure can result in crush injuries or compartment syndrome and may after ischemia, with subsequent reperfusion injury cause rhabdomyolysis and myoglobinuria. The placement of some patients in the same position for a prolonged period after surgery has also been reported to cause rhabdomyolysis, particularly in patients in an extended lithotomy position or those undergoing vascular surgeries including cases involving compartment syndrome or cardiovascular surgery ${ }^{2)}$. In the present report, we have described a fatal case wherein the placement of an obese patient in an extended prone position during spinal surgery led to rhab-

\footnotetext{
- Received: June 20, 2014 • Revised: July 28, 2014

- Accepted: August 11, 2014

Corresponding Author: Seok Won Kim, MD, PhD

Department of Neurosurgery, Chosun University College of Medicine, 365 Pilmun-daero, Dong-gu, Gwangju-city 501-717, Korea

Tel: +82-62-220-3126, Fax: +82-62-227-4575

E-mail:ns64902@hanmail.net/chosunns@chosun.ac.kr

$\otimes$ This is an Open Access article distributed under the terms of the Creative

Commons Attribution Non-Commercial License (http://creativecommons.org/ licenses/by-nc/3.0/) which permits unrestricted non-commercial use, distribution, and reproduction in any medium, provided the original work is properly cited.
}

domyolysis and acute renal failure. We also discuss the pathophysiological mechanism of this uncommon entity and review the relevant literature.

\section{CASE REPORT}

A previously healthy 29-year-old woman was referred to our institute due to paraparesis after a fall. Her visit to our institute was prompted by the development of motor weakness and voiding difficulty on the day of the visit. Physical examination revealed a weight of $118 \mathrm{~kg}$, height of $162 \mathrm{~cm}$, and body mass index of $44.9 \mathrm{~kg} / \mathrm{m}^{2}$, indicative of morbid obesity for this patient. There were no associated systemic or muscle contusions, internal organ damage, or swelling of the extremities. However, we had observed marked rigidity in both arms and legs. Preoperative laboratory test findings, including full blood count, electrolytes, creatinine, liver function test, and urine analysis were all unremarkable. However, a neurological examination revealed reduced patellar and Achilles tendon reflexes in both legs, motor weakness (Grade III/Grade III), and hypoesthesia below the T4 dermatome. Magnetic resonance imaging (MRI) and computed tomography scanning of the thoracic spine had revealed ossification of the posterior longitudinal ligament and severe stenosis at the T3, T4, and T5 levels (Fig. 1).

General anesthesia was induced using propofol, fentanyl, and rocuronium for surgery. After endotracheal intubation, the patient was placed in the prone position on the operating table cautiously, and the neck was kept in line with the spine. The head was maintained in a hyperflexion position using 
Mayfield head pins to obtain a better surgical view. Decompressive laminectomy at T4, T5 and interbody fusion at the T3-4 level by the posterior approach was performed (Fig. 2). The T3 posterior segment was removed by using drill and rongeur. Then dural sac and both T4 nerve roots were exposed. Due to the narrowing of disc space, interbody fusion using autologous block bone was performed. The surgery was uneventful and had lasted 297 minutes. After then, she was transferred to the recovery room and was alert, oriented, and hemodynamically stable. After extubation, she complained of pain in the anterior aspect of both arms and thighs. On physical examination, we noted the presence of mildly swollen and erythematous regions in her arms and thighs over the deltoid, pectoralis major, and rectus femoris muscles. Neurological examination revealed that there was no neurological change of her motor weakness (Grade III/Grade III). With the diagnosis of unsuitable positioning, she was treated with adequate hydration and analgesics for pain control. However, the color of her urine changed from clear yellow to dark brown, and myoglobinuria was developed 3 days after surgery with a high serum creatine kinase (CK) level of 38,732 IU/L (normal, 55-215 $\mathrm{IU} / \mathrm{L}$ ). Acute renal failure developed 5 days after surgery despite the initiation of intensive fluid replacement and diuresis. The CK level was peaked at 49, $735 \mathrm{IU} / \mathrm{L}$ and serum myoglobin exceeded $24 \mathrm{mh} / \mathrm{dL}$ (normal $<2.5 \mathrm{mg} / \mathrm{dL}$ ) on 5 day after surgery. She was referred to department of nephrology for intensive supportive treatment. Although the patient received intensive supportive treatment, she became anuric 7 days after admission and required hemodialysis. We could not find any evidence of muscle breakdown, but her condition had deteriorated rapidly, and she eventually had a cardiorespiratory arrest with dilated nonreactive pupils. Unfortunately, she died 12 days after spine surgery.

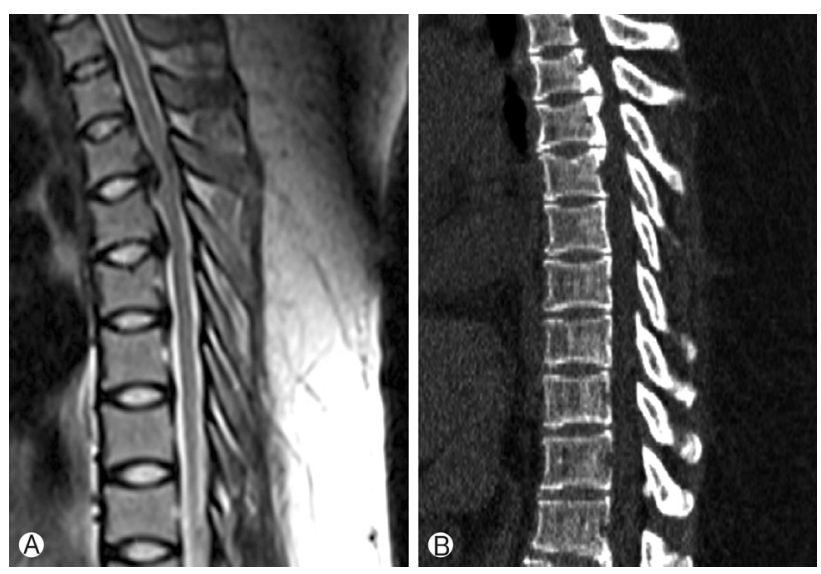

Fig. 1. T2-weighted sagittal and computed tomography scans show ossification of the posterior longitudinal ligament and severe stenosis at the T3, T4 and T5 levels.

\section{DISCUSSION}

Rhabdomyolysis, a syndrome involving skeletal muscle breakdown with leakage of muscle contents, is characterized as myalgia of acute muscle necrosis and compression palsies, leading to motor weakness ${ }^{4}$. The most common causes of rhabdomyolysis are intoxication, multisystem trauma, any other condition causing prolonged ischemia, and status epilepticus. Rhabdomyolysis after elective surgery is rare but it is possible complication after elective surgery, and is known to result from compression due to unsuitable positioning or tourniquet use. Among non-trauma patients after elective surgery, rhabdomyolysis usually occurs as a result of compression due to unsuitable positioning or tourniquet use, although a variety of additional causative factors should be considered ${ }^{5}$. Among possible causative factors, the prolonged prone position has been reported as a rare cause of rhabdomyolysis ${ }^{12)}$. In the prone position, ischemia of the muscles could result from either compression of the large vessels in the abdomen and pelvis or direct pressure on the muscle leading to hypoperfusion. Involvement of the larger blood vessels in the abdomen may also cause visceral hypoperfusion that would contribute to the elevated serum creatine phosphokinase levels ${ }^{9}$. In fact, since the posterior approach can not remove the anterior pathology, posterior approach alone can be incomplete surgery in thoracic OPLL. But, in this case, decompression of anterior lesion in the upper thoracic spine was difficult due to the sternum and presence of major vessels.

We have believed that in our morbidly obese patient, compression of the muscles caused significant rhabdomyolysis. This hypothesis was explained by the bilateral erythematous lesions in the anterior aspects of the thighs and arms. Despite

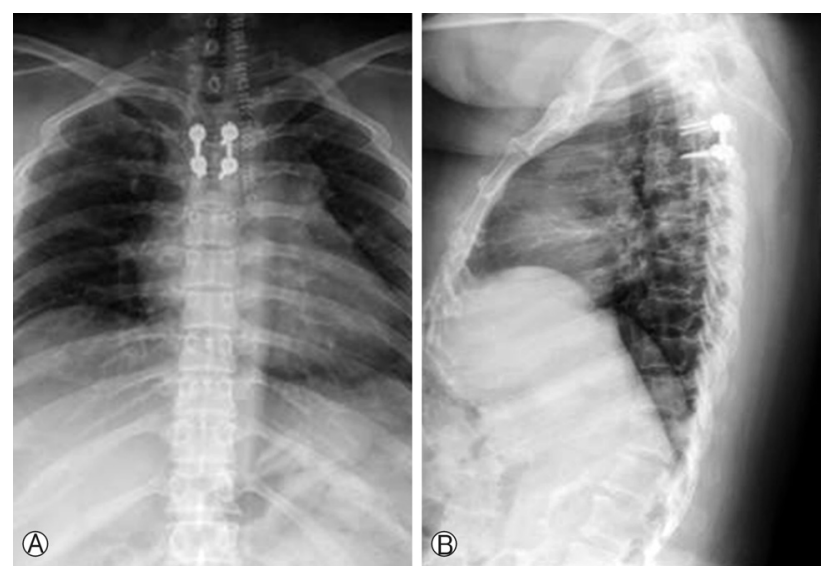

Fig. 2. Postoperative simple radiographs of the patient. 
the efforts to position this obese patient on bony prominences, particularly the iliac crest and arms, her protuberant abdomen was not fully decompressed. It is also possible that compression of the large vessels in the abdomen due to the prone position, with accompanying stasis, may have contributed to the development of rhabdomyolysis. Severe hypotension and use of a tourniquet during surgery are also considered as possible causes. However, in the present patient, no tourniquets were applied and her vital signs remained stable during surgery. The metabolism of obese patients is probably an additional risk factor to gravitational effect of obesity itself and long operation time. Although rhabdomyolysis may not always manifest with clinical signs and symptoms, it usually results in myoglobinuria and a rapid increase in creatinine phosphokinase levels, which might be its only manifestation and often goes unrecognized ${ }^{1)}$. The goal of treatment in cases of rhabdomyolysis should consist of removing the cause and preventing acute renal failure. Dialysis may be required if renal failure persists; the indications for dialysis include hyperkalemia, uremic pericarditis, and evidence of volume overload ${ }^{7}$.

\section{CONCLUSION}

We have reported a fatal case of rhabdomyolysis that developed in a morbidly obese patient who was placed in a prone position for spinal fusion surgery. Careful attention should be paid to avoid this unpredictable complication and the awareness of this special occurrence can provide early diagnosis and treatment before major spine surgery on obese patients.

\section{ACKNOWLEDGEMENT}

This study was supported by research funds from Chosun
University Hospital 2014.

\section{REFERENCES}

1. Better OS, Stein JH: Early management of shock and prophylaxis of acute renal failure in traumatic rhabdomyolysis. $\mathrm{N}$ Engl J Med 322:825-829, 1990

2. Bildsten SA, Dmochowski RR, Spindel MR, Auman JR: The risk of rhabdomyolysis and acute renal failure with the patient in the exaggerated lithotomy position. J Urol 152:1970-1972, 1994

3. Cha Kl, Kim JH, Moon BG, Kang HI, Lee SJ: Myelography induced fatal complications; seizure and rhabdomyolysis. Kor J Spine 7(3):192-194, 2010

4. Chen CM, Chen JC, Kao MC: Scvere rhabdomyolysis with good recovery in a patient with head injury: case report. Neurosurgery 41:293-296, 1997

5. Cone AM, Schneider M: Massive rhabdomyolysis following cardiopulmonary bypass. Anesth Intensive Care 23:721-724, 1995

6. Jeon HJ, Cho BM, Oh SM, Park SH: Lumbosacral plexopathy, complicating rhabdomyolysis in a 57-year-old man, presented with sudden weakness in both legs. J Korean Neurosurg Soc 42:481-483, 2007

7. Larner AJ: Rhabdomyolysis associated with cranial diabetes insipidus. Postgrad Med J 68:149, 1992

8. Park YJ, Kim SW: Fatal rhabdomyolysis in a patient with Head Injury. J Korean Neurosurg Soc 53:303-304, 2013

9. Prabhu M, Samra S: An unusual cause of rhabdomyolysis following surgery in the prone position. J Neurosurg Anesthesiol 12(4):359-356, 2000

10. Singhal PC, Abramovici M, Venkatesan J: Rhabdomyolysis in the hyperosmolar state. Am J Med 88:9-12, 1990

11. Singhal PC, Chugh KS, Gulati DR: Myoglobinuria and renal failure after status epilepticus. Neurology 28:200-201, 1978

12. Zase A, Friedhoff RJ, Rose SH: Prone position: visceral hypoperfusion and rhabdomyolysis. Anesth Analy 82:412-415, 1996 